\title{
Application of ultrasound in bone surgery: Two case reports
}

\author{
Jaume Escoda-Francolí ${ }^{1}$, Araceli Rodríguez-Rodríguez ${ }^{2}$, Leonardo Berini-Aytés ${ }^{3}$, Cosme Gay-Escoda ${ }^{4}$
}

\begin{abstract}
${ }^{1}$ DDS. Master in Oral Surgery and Implantology. University of Barcelona Dental School. Investigator of the IDIBELL Institute. Specialist of the Service of Oral surgery and Implantology of the Centro Médico Teknon. Barcelona

${ }^{2}$ DDS. Master in Oral Surgery and Implantology. University of Barcelona Dental School

${ }^{3}$ MD, DDS, PhD. Assistant Professor of Maxillofacial Surgery. Professor of the Master in Oral Surgery and Implantology. University of Barcelona Dental School. Investigator of the IDIBELL Institute

${ }^{4} \mathrm{MD}$, DDS, PhD. Chairman of Oral and Maxillofacial Surgery. Director of the Master in Oral Surgery and Implantology. University of Barcelona Dental School. Coordinating investigator of the IDIBELL Institute. Co-director of the TMJ and Orofacial Pain Unit. Head of the Service of Maxillofacial Surgery, Teknon Medical Center. Barcelona, Spain
\end{abstract}

Correspondence:

Centro Médico Teknon

Instituto de Investigación IDIBELL

Vilana 12

08022 - Barcelona (Spain)

cgay@ub.edu

Received: 01/10/2009

Accepted: 23/04/2010

Escoda-Francolí J, Rodríguez-Rodríguez A, Berini-Aytés L, Gay-Escoda C. Application of ultrasound in bone surgery: Two case reports. Med Oral Patol Oral Cir Bucal. 2010 Nov 1;15 (6):e902-5.

http://www.medicinaoral.com/medoralfree01/v15i6/medoralv15i6p902.pdf

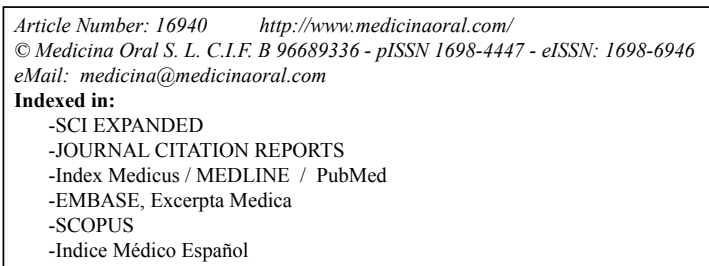

\begin{abstract}
The present study describes some of the applications of ultrasound in bone surgery, based on the presentation of two clinical cases.

The Piezosurgery ${ }^{\circledR}$ ultrasound device was used (Tecnología Mectron Medical, Carasco, Italy). In one case the instrument was used to harvest a chin bone graft for placement in a bone defect at level 1.2, while in the other case a bony window osteotomy was made in the external wall of the maxillary sinus, in the context of a sinus membrane lift procedure.

The Piezosurgery ${ }^{\circledR}$ device produces specific ultrasound frequency modulation $(25-29 \mathrm{kHz})$, and has been designed to secure increased precision in application to bone surgery. This instrument produces selective sectioning of the mineralized bone structures, and causes less intra- and postoperative bleeding.

One of the advantages of the Piezosurgery ${ }^{\circledR}$ device is that it can be used for maxillary sinus lift procedures in dental implant placement. In this context it considerably lessens the risk of sinus mucosa laceration by preparing the bony window in the external wall of the upper maxilla, and can be used to complete the lifting maneuver.

The use of ultrasound in application to hard tissues can be regarded as a slow technique compared with the conventional rotary instruments, since it requires special surgical skill and involves a certain learning curve.
\end{abstract}

Key words: Bone surgery with ultrasound, Piezosurgery®. 


\section{Introduction}

Kennedy et al. (1) reported that Pohlman in 1950 was the first to apply ultrasound in humans, on an empirical basis for the treatment of neuropathic pain and myalgias. The therapeutic application of ultrasound subsequently became increasingly widespread, on the belief that practically any disorder could benefit from the technique. However, its application to bone was not advocated until 1950, when Maintz published the first study pointing out the beneficial effects of ultrasound upon bone healing (2).

Ultrasound was first applied in dentistry in 1952, specifically for preparing dental cavities, though in this context the technique was subsequently displaced by the introduction of high-speed rotary instruments. Nevertheless, its applications in other areas gradually increased over the years.

Piezosurgery ${ }^{\circledR}$ is an ultrasound device introduced in medical practice in 1988 for different procedures in application to hard tissues, including periodontal surgery, periapical surgery $(3,4)$, the removal of impacted teeth, implant surgery for facilitating bone ridge expansion or in bone regeneration techniques $(5,6)$, inferior dental nerve lateralization and transpositioning - thanks to the fact that the device does not damage non-mineralized structures (7) - and in different interventions such as orthognathic surgery. The Piezosurgery ${ }^{\circledR}$ system is equipped with several ultrasound tips specific for each type of procedure (5).

The instrument used in our study operates at a frequency of $25-29 \mathrm{kHz}$, with an advanced oscillation control module that introduces pauses in the high-frequency vibrations. These pauses help avoid heating of the bone and maintain optimum sectioning capacity. The amplitude of the microvibrations is in the range of $60-210 \mu \mathrm{m}$, corresponding to a handpiece power rating of over $5 \mathrm{~W}$ (8).

This system was developed with the purpose of securing improved precision and safety in bone surgery compared with the manual and rotary instruments.

The Piezosurgery ${ }^{\circledR}$ device offers three specific features attributable to the microvibrations and to specific modulation of the ultrasound frequency $(25-29 \mathrm{kHz})$. Firstly, it allows micrometric sectioning, offering superior precision in cutting and with no bone loss. Secondly, the instrument selectively sections mineralized structures, without damaging the adjacent soft tissues, which remain intact even in the case of accidental contact with the device. Lastly, the physical cavitation phenomenon produced by the device ensures less bleeding (9).

\section{Clinical Cases}

\section{Case 1}

A 23-year-old woman presented with no disease antecedents of interest and no known drug allergies. She re- ported occasional alcohol consumption and no smoking. Surgery for ectopic pregnancy had been carried out one year ago. The patient was seen for en bloc bone graft treatment of absent tooth 1.2, since the area presented a horizontal bony defect, with posterior implant placement (Figure 1A). She had a removable dental prosthesis in position 1.2. Moderate generalized periodontal disease (as established by the CPI index) was observed, with good oral hygiene (plaque index). The patient was cooperative but nervous. Initial management consisted of periodontal treatment. Posteriorly an en bloc chin bone graft was harvested, using the Piezosurgeryy instrument in advanced boosted mode with external irrigation and the ultrasound tip specifically designed for this procedure (Figure 1B). Surgery was carried out under local anesthesia and conscious sedation via the intravenous route (midazolam, propofol and remifentanil). The graft was affixed to the zone of the defect with a titanium screw (Figure 1C). Antibiotics and analgesics were provided in the postoperative period: amoxicillin clavulanic acid 875 / $125 \mathrm{mg}$ every 8 hours for 10 days, and diclofenac $50 \mathrm{mg}$ every 8 hours for 5 days. In second step surgery 7 months later, the screw was removed

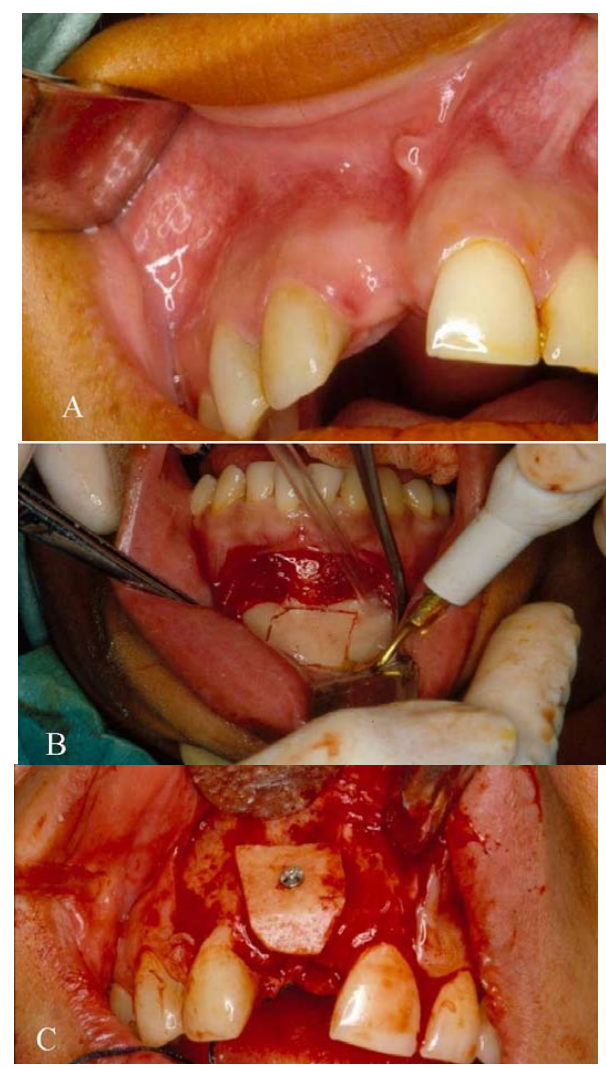

Fig. 1. Case 1.

(A) Clinical view showing the bone defect in the zone of 1.2 .

(B) Harvesting of the chin graft.

(C) Fixation of the graft to the defect zone. 
and a palatal keratinized gingival graft was positioned. Two months later a dental implant was placed in the zone, followed by prosthetic rehabilitation. The esthetic outcome can be considered acceptable.

\section{Case 2}

A 43-year-old woman presented with no disease antecedents of interest and no known drug allergies. She had stopped smoking a year ago, and reported moderate alcohol consumption. The patient had undergone tonsillectomy 15 years ago; she showed good general health and was cooperative.

The patient was seen in our center for implant-supported rehabilitation of edentulous spaces. Examination revealed posterior sector atrophy of the upper maxilla. She wore no dentures. Her periodontal condition was adequate, with good oral and dental hygiene. The fol-

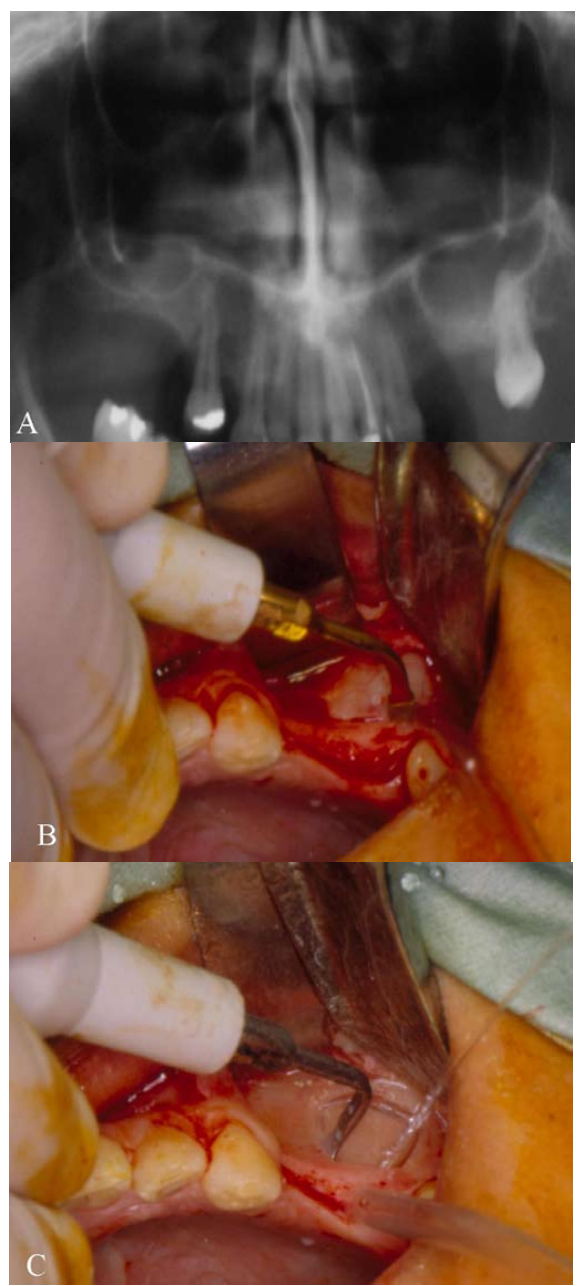

Fig. 2. Case 2.

(A) Panoramic X-ray view showing important alveolar atrophy, with pneumatization of both maxillary sinuses.

(B) Osteotomy for preparing the bony window giving access to the left maxillary sinus.

(C) Dissection of the sinus membrane. lowing teeth were missing: $1.8,1.7,1,6,1.5,2.4,2.5,2.6$, $3.8,3.7,3.6,3.5$ and 4.6. The radiological study revealed bone atrophy of the upper maxilla, with considerable pneumatization of both maxillary sinuses (Figure 2A). The treatment strategy consisted of bone regeneration in the posterior sector of the upper maxilla, with bilateral maxillary sinus lifting, followed by implant-supported rehabilitation. The Piezosurgery ${ }^{\circledR}$ instrument was used in advanced boosted mode with external irrigation to prepare the bony windows and lift the sinus membrane. These procedures were carried out using the ultrasound tips recommended by the manufacturer (Figures 2B and $2 \mathrm{C}$ ). There was no perforation of the sinus membrane, and the bony window was lifted with the membrane, conforming the new floor of the maxillary sinus. The resulting space was filled with a 50\% mixture of crushed autologous bone harvested from the chin and Bio-Oss ${ }^{\circledR}$ (Geisttich Pharma, Wolhusen, Switzerland), covering the entire surgical zone with a reabsorbable type I collagen membrane (Biomend $\AA$, Zimmer Dental, New Jersey, USA). The same medication as in the previous case was prescribed, and the postoperative course was free of complications. Prosthesis over implants rehabilitation proved satisfactory.

\section{Discussion}

Piezoelectric surgery applied to bone, also known as the Piezosurgery ${ }^{\circledR}$ technique, is a new option for osteotomies and ostectomies, using an ultrasound device.

In 1975, Horton et al. (10) compared alveolar bone healing in surgical defects produced by ultrasound with defects produced using a chisel or rotary instruments good results being obtained with all three techniques. In 1981 these same authors published another study on the clinical applications of ultrasound in bone surgery (11). Posteriorly, an experimental study in dogs was published, comparing bone healing using the Piezosurgery ${ }^{\circledR}$ device, conventional tungsten carbide drills or diamond drills to create 4-mm bone defects around the posterior mandibular teeth. The authors concluded that the Piezosurgery ${ }^{\circledR}$ device offered the best results in terms of bone regeneration (12).

The sinus membrane lift technique implies the risk of perforating the Schneider membrane. This relatively common complication can be caused by the heat generated during osteotomy, drill action on creating the bony window, or on lifting the membrane with manual dissectors. One of the advantages of the Piezosurgery ${ }^{\circledR}$ device is that it can be used to prepare the bony window in the external wall of the upper maxilla without the risk of lacerating the sinus mucosa, and with a limited loss of bone tissue. Vercellotti et al. (13) evaluated 15 patients operated upon with the Piezosurgery ${ }^{\circledR}$ device in the context of bone regeneration in pneumatized maxillary sinuses. A total of 21 osteotomies were carried out 
for bony window preparation, with a mean length of 14 $\mathrm{mm}$, a height of $6 \mathrm{~mm}$ and a thickness of $1.4 \mathrm{~mm}$. The mean duration of bony window preparation was three minutes. Posterior sinus membrane lift with this instrument required 5 minutes on average. The reported success rate was $95 \%$, with a single sinus membrane perforation on performing the osteotomy.

Autologous bone grafts are widely used in implant surgery to secure horizontal or vertical alveolar process augmentation in atrophic maxillas. Chiriac et al. (14) compared the viability, growth and cell differentiation of bone grafts harvested with a piezoelectric device (Piezosurgery $\left.{ }^{\circledR}\right)$ or a conventional rotary instrument. The authors concluded that neither system interfered with joining of the bone grafts to the recipient bone bed. We obtained satisfactory results with the Piezosurgery ${ }^{\circledR}$ device in harvesting bone grafts, with subsequent correct healing in the recipient bed.

In application to other procedures such as residual crest expansion for posterior implant placement, ultrasound has been shown to be fundamental for ensuring precise yet minimally traumatic surgery (15).

Lastly, it should be mentioned that the Piezosurgery ${ }^{\circledR}$ system constitutes an innovation in bone surgery, with handling characteristics that are different from those of the conventional techniques. In effect, it requires special surgical skill and involves a certain learning curve (1).

\section{References}

1. Kennedy JE, Ter Haar GR, Cranston D. High intensity focused ultrasound: surgery of the future? Br J Radiol. 2003;76:590-9.

2. Maintz G. [Animal experiments in the study of the effect of ultrasonic waves on bone regeneration.]. Strahlentherapie. 1950;82:631-8. 3. Peñarrocha Diago M, Ortega Sánchez B, García Mira B, Martí Bowen E, von Arx T, Gay Escoda C. Evaluation of healing criteria for success after periapical surgery. Med Oral Patol Oral Cir Bucal. 2008;13:E143-7.

4. Martí-Bowen E, Peñarrocha-Diago M, García-Mira B. Periapical surgery using the ultrasound technique and silver amalgam retrograde filling. A study of 71 teeth with 100 canals. Med Oral Patol Oral Cir Bucal. 2005;10 Suppl 1:E67-73.

5. Vercellotti T. Technological characteristics and clinical indications of piezoelectric bone surgery. Minerva Stomatol. 2004;53:207-14.

6. Eggers G, Klein J, Blank J, Hassfeld S. Piezosurgery: an ultrasound device for cutting bone and its use and limitations in maxillofacial surgery. Br J Oral Maxillofac Surg. 2004;42:451-3.

7. Metzger MC, Bormann KH, Schoen R, Gellrich NC, Schmelzeisen R. Inferior alveolar nerve transposition--an in vitro comparison between piezosurgery and conventional bur use. J Oral Implantol. 2006;32:19-25.

8. Schlee M, Steigmann M, Bratu E, Garg AK. Piezosurgery: basics and possibilities. Implant Dent. 2006;15:334-40.

9. Stübinger S, Kuttenberger J, Filippi A, Sader R, Zeilhofer HF. Intraoral piezosurgery: preliminary results of a new technique. J Oral Maxillofac Surg. 2005;63:1283-7.

10. Horton JE, Tarpley TM Jr, Wood LD. The healing of surgical defects in alveolar bone produced with ultrasonic instrumentation, chisel, and rotary bur. Oral Surg Oral Med Oral Pathol. 1975;39:53646.
11. Horton JE, Tarpley TM Jr, Jacoway JR. Clinical applications of ultrasonic instrumentation in the surgical removal of bone. Oral Surg Oral Med Oral Pathol. 1981;51:236-42.

12. Vercellotti T, Nevins ML, Kim DM, Nevins M, Wada K, Schenk $\mathrm{RK}$, et al. Osseous response following resective therapy with piezosurgery. Int J Periodontics Restorative Dent. 2005;25:543-9.

13. Vercellotti T, De Paoli S, Nevins M. The piezoelectric bony window osteotomy and sinus membrane elevation: introduction of a new technique for simplification of the sinus augmentation procedure. Int J Periodontics Restorative Dent. 2001;21:561-7.

14. Chiriac G, Herten M, Schwarz F, Rothamel D, Becker J. Autogenous bone chips: influence of a new piezoelectric device (Piezosurgery) on chip morphology, cell viability and differentiation. J Clin Periodontol. 2005;32:994-9.

15. Danza M, Guidi R, Carinci F. Comparison between implants inserted into piezo split and unsplit alveolar crests. J Oral Maxillofac Surg. 2009;67:2460-5.

\section{Acknowledgements}

This study has been carried out by the consolidated research group in "Dental and Maxillofacial Pathology and Treatment" of the Institut d'Investigació Biomèdica de Bellvitge (IDIBELL), with financial support from the oral surgery teaching-healthcare agreement among the University of Barcelona, the Consorci Sanitari Integral and the Servei Català de la Salut of the Generalitat de Catalunya. 